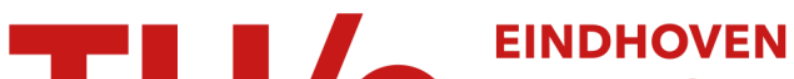 \\ UNIVERSITY OF \\ TECHNOLOGY
}

\section{Solution concepts for hybrid dynamical systems}

\section{Citation for published version (APA):}

Camlibel, M. K., Heemels, W. P. M. H., Schaft, van der, A. J., \& Schumacher, J. M. (2003). Solution concepts for hybrid dynamical systems. In E. F. Camacho, \& L. Basanez (Eds.), Proceedings of the 15th Triennial World Congress of the International Federation of Automatic Control(IFAC202), 21-26 June 2002, Barcelona, Spain [1217] Pergamon.

\section{Document status and date:}

Published: 01/01/2003

\section{Document Version:}

Publisher's PDF, also known as Version of Record (includes final page, issue and volume numbers)

\section{Please check the document version of this publication:}

- A submitted manuscript is the version of the article upon submission and before peer-review. There can be important differences between the submitted version and the official published version of record. People interested in the research are advised to contact the author for the final version of the publication, or visit the $\mathrm{DOI}$ to the publisher's website.

- The final author version and the galley proof are versions of the publication after peer review.

- The final published version features the final layout of the paper including the volume, issue and page numbers.

Link to publication

\section{General rights}

Copyright and moral rights for the publications made accessible in the public portal are retained by the authors and/or other copyright owners and it is a condition of accessing publications that users recognise and abide by the legal requirements associated with these rights.

- Users may download and print one copy of any publication from the public portal for the purpose of private study or research.

- You may not further distribute the material or use it for any profit-making activity or commercial gain

- You may freely distribute the URL identifying the publication in the public portal.

If the publication is distributed under the terms of Article $25 \mathrm{fa}$ of the Dutch Copyright Act, indicated by the "Taverne" license above, please follow below link for the End User Agreement:

www.tue.nl/taverne

Take down policy

If you believe that this document breaches copyright please contact us at:

openaccess@tue.nl

providing details and we will investigate your claim. 


\title{
SOLUTION CONCEPTS FOR HYBRID DYNAMICAL SYSTEMS
}

\author{
M.K. Çamlıbel ${ }^{*}$ W.P.M.H. Heemels ${ }^{\dagger}$ A.J. van der Schaft ${ }^{\dagger}$ \\ J.M. Schumacher ${ }^{\S}$
}

\author{
* Dept. Math., Groningen Univ., P.O. Box 800, 9700 AV Groningen. \\ k. camlibel @math.rug.nl \\ ${ }^{\dagger}$ Dept. Electr. Eng., Eindhoven Univ. Technology, P.O. Box 513, 5600 \\ MB Eindhoven. w.p.m.h.heemels d tue.nl \\ * Fac. Math. Sciences, Twente Univ., P.O. Box 217, Enschede. \\ a.j.vanderschaft@math. utwente.nl \\ $\S$ Dept. Econometrics and Oper. Research, Tilburg University, P.O. Box \\ 90153, 5000 LE Tilburg. j.m. schumacher@kub.nl
}

\begin{abstract}
The recent interest in hybrid systems has given rise to a large variety of model classes and to many different notions of solution trajectories. In this paper we enumerate several solution concepts and compare them on the basis of some examples displaying Zeno behaviour. The relation to well-posedness is also discussed.
\end{abstract}

Keywords: Hybrid systems, solution concepts, well-posedness, Zeno behaviour.

\section{INTRODUCTION}

Very broadly speaking, scientific modeling may be defined as the process of finding common descriptions for groups of observed phenomena. Often, several description forms are possible. In system and control theory differential equations are often used. Such descriptions may be viewed as being fairly indirect; after all it represents trajectories only as solutions to some problem, rather than expressing directly what the trajectories are. There are many examples in science where, as above, an implicit description (that is, a description in terms of a mathematical problem that needs to be solved) is useful and possibly more powerful than explicit descriptions. Whenever an implicit description is used, however, one has to show that the description is a "good" one in the sense that the stated problem has a well-defined solution. This is essentially the issue of well-posedness. Typically, for differential equations it is required that solutions exist and are unique for any given initial condition. Both for the existence and for the uniqueness statement, one has to specify a function class in which solutions are considered. The broader the function class is, the easier existence of trajectories is guaranteed and the more difficult it is to have uniqueness of trajectories. Hence, there is a clear interaction between the chosen solution concept and well-posedness.

In this article we will compare several solution concepts being around in the literature for hybrid dynamical systems. Many different description formats have been pro- posed in recent years. In the discrepancy between the concepts a crucial role is played by the particular types of admissible Zeno behaviour, i.e. the phenomenon that an infinite number of discrete events occur in a finite length time interval. A motivation for looking at Zeno solutions may be derived from the following water tank example (Alur and Henzinger, 1997). Consider the system

$$
\dot{x}_{1}=-\epsilon+u ; \dot{x}_{2}=-\epsilon+(1-u),
$$

where $\epsilon$ is a constant between $\frac{1}{2}$ and 1 and where $u$ is a hybrid control defined as follows: if $x_{1}=0$ and $x_{2}>0$ then the control switches to $u=1$, if $x_{2}=0$ and $x_{1}>0$ then the control switches to $u=0$. The idea is that we have a pump that tries to prevent the tanks from running empty; but the tanks are leaking with a total outflow $2 \varepsilon$ larger than the inflow of 1 and so there's no ultimate cure. Nevertheless, one can show that the goal is achieved along all non-Zeno trajectories.

This example shows that it is crucial to incorporate Zenoness in the study of hybrid systems (i.e. in the solution concept) to prevent that wrong conclusions are drawn. Since the inclusion of Zeno behaviour leads to a substantial increase of the complexity of the analysis, conditions excluding Zenoness would be very practical and welcome, but unfortunately verifiable conditions are hardly ever presented. In this paper we will also discuss the difficulties caused by Zeno behaviour in the study of well-posedness. 


\section{MODEL CLASSES}

We will start by presenting certain model classes that have been adopted for continuous-time ${ }^{1}$ hybrid systems and for which well-posedness issues have been studied.

\subsection{The hybrid automaton model}

A useful framework to describe hybrid dynamical systems is that of a hybrid automaton, see (Alur et al., 1995; Lynch et al., 1996). Basically, a hybrid automaton merges the standard concepts of automata and continuous-time dynamics, by modeling the discrete part of the dynamics by means of a graph whose vertices are called locations and whose edges are transitions. To every discrete state or location $\ell \in$ Loc of the automaton one associates a continuous-time dynamics ${ }^{2}$ $\dot{x}=f_{\ell}(x)$ governing the evolution of the continuous state $x$. Interaction between the discrete dynamics and the continuous dynamics takes place through invariants and transition relations. Indeed, the continuoustime dynamics may induce discrete transitions in the locations by specifying for every location $\ell$ a so-called location invariant $\operatorname{Inv}(\ell)$, which is a subset of the continuous state space $X=\mathbb{R}^{n}$, specifying the feasible set of continuous states for the location $\ell$, in the sense that if exit of the continuous state from the location invariant is imminent, then a transition to another location $\ell^{\prime}$ and / or a reset of the continuous state $x$ has to take place (or the system is in a deadlock). The discrete transitions are given by a collection of edges $E \subset \operatorname{Loc} \times$ Loc. For every discrete transition $\left(\ell, \ell^{\prime}\right) \in E$ a guard $G\left(\ell, \ell^{\prime}\right) \subset X$ is specified, defining enabling conditions on the continuous state in order that the transition to $\ell^{\prime}$ may take place. Another interplay between discrete and continuous dynamics is provided by the reset relations $R\left(\ell, \ell^{\prime}\right) \subset X \times X$, specifying for every discrete transition $\left(\ell, \ell^{\prime}\right) \in E$ the continuous state reset from $x \in G\left(\ell, \ell^{\prime}\right)$ to $x^{\prime} \in X$ such that $\left(x, x^{\prime}\right) \in R\left(\ell, \ell^{\prime}\right)$. Sometimes a set of initial (hybrid) states Init $\subseteq$ Loc $\times X$ is given.

\subsection{Discontinuous differential equations}

For a typical example of differential equations with discontinuous right hand sides (Filippov, 1988; Utkin, 1981), consider:

$$
\begin{aligned}
& \dot{x}=f_{1}(x) \quad(h(x)>0) \\
& \dot{x}=f_{2}(x) \quad(h(x)<0),
\end{aligned}
$$

where $h$ is a real-valued function. The specification above is incomplete as no statement is made for $h(x)=$ 0 . One way to arrive at a solution concept is to adopt a suitable relaxation. Specifically, in a convex relaxation one would rewrite the equations (1) as $\dot{x} \in F(x)$, where the set-valued function $F(x)$ is defined by

$F(x)=\left\{f_{1}(x)\right\},(h(x)>0)$

$F(x)=\left\{f_{2}(x)(h(x)<0)\right.$,

$F(x)=\left\{a f_{1}(x)+(1-a) f_{2}(x) \mid a \in[0,1]\right\}(h(x)=0)$,

where it is assumed that $f_{1}$ and $f_{2}$ are continuous functions defined on $\{x \mid h(x) \geq 0\}$ and $\{x \mid h(x) \leq$

\footnotetext{
1 The restriction to continuous-time is taken as the interaction between discrete and continuous dynamics gives much more interesting questions (e.g. related to Zenoness) than for the discrete-time case. For discrete-time systems well-posedness usually comes down to solvability properties of algebraic problems.

2 In a more general setting these might also be specified by differential and algebraic equations $F_{\ell}(x, \dot{x})=0$.
}

$0\}$, respectively. Now a differential inclusion has been obtained, and corresponding solution concepts can be applied (Aubin and Cellina, 1984). Other methods to obtain differential inclusions are proposed by Utkin ('control equivalent definition') and Aizerman and Pyatnitskii (see (Filippov, 1988) for an overview).

\subsection{Piecewise or multi-modal linear systems}

As a subclass of the systems of the previous subsection, consider multi-modal or piecewise linear systems of the form

$$
\dot{x}=A_{i} x, \text { if } x \in \mathcal{C}_{i}, i=1, \ldots, r,
$$

where $\mathcal{C}_{i}$ are certain subsets of $\mathbb{R}^{n}$ with

$$
\bigcup_{i=1}^{r} \mathcal{C}_{i}=\mathbb{R}^{n} \text { and int } \mathcal{C}_{i} \cap \operatorname{int} \mathcal{C}_{j}=\emptyset, \quad i \neq j
$$

\subsection{Complementarity systems}

Systems of the form (1) are sometimes known as variable-structure systems; they describe a type of mode-switching. A similar mode-switching behavior is obtained from a class of systems known as complementarity systems (van der Schaft and Schumacher, 1998; Heemels et al., 2000). Equations for a complementarity system may be written in terms of a state variable $x$ and auxiliary variables $v$ and $z$, which must be vectors of the same length, as:

$$
\begin{aligned}
& \dot{x}=f(x, v) \\
& z=h(x, v) \\
& 0 \leq z \perp v \geq 0,
\end{aligned}
$$

where the last line means that the components of the auxiliary variables $v$ and $z$ should be nonnegative, and that for each index $i$ and for each time $t$ at least one of the two variables $v_{i}(t)$ and $z_{i}(t)$ should be equal to 0 . The description (4) is in principle implicit in the discrete variables or modes. However, like (1), the system (4) can be considered to consist of a number of different dynamical systems or "modes" that are glued together. The modes can be thought of as discrete states. They correspond to a fixed choice, for each of the indices $i$, between the two possibilities $v_{i} \geq 0, z_{i}=0$ and $v_{i}=0, z_{i} \geq 0$, so that a complementarity system in which the vectors $v$ and $z$ have length $m$ has $2^{m}$ different modes. The specification (4) is in general not complete yet; one has to add a rule that describes possible jumps of the state variable $x$ when a transition from one mode to another takes place (think of mechanical systems with impacts). In principle, this forms a distinction with the previous two subsections as the state trajectories are continuous in those cases. The complementarity system can in principle be rewritten in the explicit hybrid automaton format, but the representation that is obtained may be awkward (see (Heemels et al., 1999)).

\section{SOLUTION CONCEPTS}

A description format for a class of dynamical systems only specifies a collection of trajectories if one provides a notion of solution. Actually the term "solution" already more or less suggests an implicit description format; in computer science terms, one may also say that a definition should be given of what is understood by a run (or an execution). Formally speaking, description formats are a matter of syntax: they specify what is a well-formed expression. The notion of solution 
provides semantics: to each well-formed expression it associates a collection of functions of time. Here we review solution concepts for several of the description formats that were introduced.

\subsection{Hybrid automata}

To present the solution concept for hybrid automata, we first define "hybrid time trajectories."

Definition 3.1. (Johansson et al., 1999b) A hybrid time trajectory $\tau=\left\{I_{i}\right\}_{i=0}^{N}$ is a finite $(N<\infty)$ or infinite $(N=\infty)$ sequence of intervals of the real line, such that

- $I_{i}=\left[\tau_{i}, \tau_{i}^{\prime}\right]$ with $\tau_{i} \leq \tau_{i}^{\prime}=\tau_{i+1}$ for $0 \leq i<N$;

- if $N<\infty$, either $I_{N}=\left[\tau_{N}, \tau_{N}^{\prime}\right]$ or $I_{N}=$ $\left[\tau_{N}, \tau_{N}^{\prime}\right)$ with $\tau_{N} \leq \tau_{N}^{\prime} \leq \infty$.

Definition 3.2. An execution $\chi$ of a hybrid automaton is a collection $\chi=(\tau, \lambda, \xi)$ with $\tau$ a hybrid time trajectory, $\lambda: \tau \rightarrow$ Loc and $x: \tau \rightarrow X$, satisfying

- $\left(\lambda\left(\tau_{0}\right), \xi\left(\tau_{0}\right)\right) \in$ Init (initial condition);

- for all $i$ such that $\tau_{i}<\tau_{i}^{\prime}, \xi$ is continuously differentiable and $\lambda$ is constant on $\left[\tau_{i}, \tau_{i}^{\prime}\right]$, and $\xi(t) \in \operatorname{Inv}(\lambda(t))$ and $\dot{\xi}(t)=f_{\lambda(t)}(\xi(t))$ for all $t \in\left[\tau_{i}, \tau_{i}^{\prime}\right)$ (continuous evolution); and

- for all $i, e=\left(\lambda\left(\tau_{i}^{\prime}\right), \lambda\left(\tau_{i+1}\right)\right) \in E, \xi\left(\tau_{i}^{\prime}\right) \in G(e)$ and $\left(x\left(\tau_{i}^{\prime}\right), x\left(\tau_{i+1}\right)\right) \in R(e)$ (discrete evolution).

\subsection{Discontinuous differential equations}

As seen above, some hybrid systems can be viewed as differential inclusions with the following standard solution concept.

Definition 3.3. A function $x:[a, b] \mapsto \mathbb{R}^{n}$ is a solution of $\dot{x} \in F(x)$, if $x$ is absolutely continuous and satisfies $\dot{x}(t) \in F(x(t))$ for almost all $t \in[a, b]$.

An alternative solution concept for $\dot{x} \in F(t, x)$ can be formed by taking limits of approximate solutions defined by some approximation scheme ("sampling solutions" (Clarke et al., 1997) and "Euler solutions" (Clarke et al., 1998)). This concept is based on taking a selection $f$ of $F$, i.e. a function $f$ such that $f(t, x) \in$ $F(t, x)$ for all $(t, x)$ (Clarke et al., 1998). Then a generalized solution concept is used for the differential equation $\dot{x}=f(t, x)$, which does not require any particular regularity of $f$.

Given initial state $x(0)=x_{0}$ an approximation on the interval $[0, T]$ is made by selecting a set of discretization points $\pi=\left\{t_{0}, t_{1}, \ldots, t_{N}\right\}$ with $t_{0}=0$ and $t_{N}=T$. The mesh size of this set is defined by $\mu_{\pi}:=\max \left\{t_{i}-t_{i-1} \mid 1 \leq i \leq N\right\}$. Given this set of discretization points we obtain a piecewise linear function $x_{\pi}$ by applying the Euler integration routine:

$$
x_{\pi}\left(t_{i+1}\right)=x_{\pi}\left(t_{i}\right)+\left(t_{i+1}-t_{i}\right) f\left(x_{\pi}\left(t_{i}\right), t_{i}\right)
$$

for $i=0, \ldots, N-1, x_{\pi}\left(t_{0}\right)=x_{0}$ and using linear interpolation between the discretization points. Such a piecewise linear approximation is called an Euler polygonal arc. A solution to $\dot{x}=f(t, x)$ with initial state $x_{0}$ is a uniform limit of some sequence of Euler polygonal arcs $x_{\pi_{j}}$ with $\mu_{\pi_{j}} \rightarrow 0$. An Euler solution of $\dot{x} \in F(t, x)$ with initial state $x_{0}$ is now defined as an Euler solution to $\dot{x}=f(t, x)$ with initial state $x_{0}$, where $f$ is some selection of $F$.

\subsection{Multimodal linear systems}

The solution concept that is employed for (2) in (Imura and van der Schaft, 2000) is the extended Carathéodory solution, which is based on considering the differential equations $\dot{x}=f(x)$ with $f(x)$ the (discontinuous) vector field given by the right-hand side of (2).

Definition 3.4. The function $x:\left[t_{0}, t_{1}\right] \mapsto \mathbb{R}^{n}$ is an extended Carathéodory solution, if $x$ is absolutely continuous on $\left[t_{0}, t_{1}\right]$, satisfies

$$
x(t)=x\left(t_{0}\right)+\int_{t_{0}}^{t} f(x(\tau)) d \tau,
$$

and if there are no left-accumulation points in the set of event times ${ }^{3}$, i.e. the collection of time instants at which switches are made from $A_{i} x$ to $A_{j} x$ for some $i \neq j$.

\subsection{Complementarity systems}

For complementarity systems one may develop several solution concepts, which may be similar to the notion of an execution for hybrid automata, or to the solution concept for differential inclusions as discussed above. A solution concept of the first type can for instance be formulated as follows.

Definition 3.5. A triple $(v, x, z)$ of vector functions is said to be a forward solution of the system (4) on the interval $[a, b)$, if $x$ is continuous, there exists a sequence of time points $\left(t_{0}, t_{1}, \ldots\right)$ with $t_{0}=a, t_{j+1}>t_{j}$ for all $j$, and either $t_{N}=b$ or $\lim _{j \rightarrow \infty} t_{j}=b$, as well as for each $j=0,1, \ldots$ an index set $I_{j}$, such that for all $j$ the restrictions of $x(\cdot), v(\cdot)$, and $z(\cdot)$ to $\left(t_{j}, t_{j+1}\right)$ are real-analytic, and for all $t \in\left(t_{j}, t_{j+1}\right)$ the following holds:

$$
\begin{aligned}
& \dot{x}(t)=f(x(t), v(t)), z(t)=h(x(t), v(t)) \\
& z_{i}(t)=0 \text { for } i \in I_{j}, v_{i}(t)=0 \text { for } i \notin I_{j} \\
& z_{i}(t) \geq 0 \text { for } i \notin I_{j}, v_{i}(t) \geq 0 \text { for } i \in I_{j} .
\end{aligned}
$$

The definition requires that the $x$-part of the solution is continuous across events. For so-called "high-index" systems (e.g. unilaterally constrained mechanical systems), this requirement is too strong and one has to add jump rules that connect continuous states before and after an event has taken place. Under suitable conditions, a general jump rule may be given; see (Heemels et al., 2000; van der Schaft and Schumacher, 1998). Another possibly restrictive aspect of the definition lies in the fact that it assumes that the set of event times is well-ordered ${ }^{4}$ by the usual order of the reals, but not necessarily by the reverse order; in other words, event times may accumulate to the right, but not to the left. This lack of symmetry with respect to time can be removed by allowing the set of event times $\&$ to be of a more general type. For instance, one may require that $\mathscr{E}$ is closed and nowhere dense; ${ }^{5}$ this guarantees that the complement of $\varepsilon$ is open and that for each event time $\tau$ one can construct sequences of non-event

\footnotetext{
3 An element $t$ of a set $\mathcal{E}$ is said to be a left (right) accumulation point if for all $t^{\prime}>t\left(t^{\prime}<t\right)\left(t, t^{\prime}\right) \cap \mathcal{E}\left(\left(t^{\prime}, t\right) \cap \mathcal{E}\right)$ is not empty.

4 An ordered set $S$ is said to be well-ordered if each nonempty subset of $S$ has a least element.

${ }^{5}$ A closed subset of a topological space is nowhere dense if and only if its interior is empty.
} 
times converging to $\tau$, both of which may be useful properties for other parts of the definition. Indeed, at a right-accumulation point of event times one can try to construct the left-limit of the state $x$ and to continue from this limit state in 'forward time.' In the setting of complementarity or multi-modal linear system this is possible as the discrete state is in principle determined by the continuous state. Hence, this provides a means to define a 'natural relaxation' of these discontinuous dynamical systems to arrive at solutions beyond Zeno times. See for instance, the three-ball example and Filippov's example below. Solutions that are obtained in this way are called hybrid solutions, because the corresponding solution concept is still based on explicit reference to event times.

An alternative concept that foregoes explicit mention of events is the following one.

Definition 3.6. A triple $(x, v, z) \in L_{2}^{n+2 m}$ is said to be an $L_{2}$-solution of (4) on the interval $[0, T]$ with initial condition $x_{0}$ if for almost all $t \in[a, b]$ the following conditions hold:

$$
\begin{aligned}
& x(t)=x_{0}+\int_{0}^{t} f(x(s), v(s)) d s \\
& z(t)=h(x(t), v(t)) \\
& 0 \leq z(t) \perp v(t) \geq 0 .
\end{aligned}
$$

This definition is in the spirit of the one given above for differential inclusions like Filippov's solutions ('convex definition) or Utkin's solutions ('control equivalent definition').

\section{WELL-POSEDNESS}

Well-posedness roughly means that solutions exist and are unique for any given initial condition. Both for the existence and for the uniqueness statement, one has to specify a function class in which solutions are considered. The function class used for existence may be the same as the one used for uniqueness, or it may be different. If solutions exist and are unique, a given system description defines a mapping from initial conditions to trajectories. In the theory of smooth dynamical systems, it is usually taken as part of the definition of wellposedness that this mapping is continuous with respect to suitably chosen topologies. This may be a too strong requirement for hybrid systems.

Besides the function classes ('solution concepts') considered, one may also distinguish between various notions of well-posedness on the basis of the time interval that is involved. For instance, in the context of hybrid automata, one may say that a given automaton is nonblocking (Johansson et al., 1999b), if for each initial condition either at least one transition is enabled or a smooth continuation within one of the locations during an interval of positive length is possible. Alternatively, this type of well-posedness can be described by saying that "deadlock is absent." If the continuation is unique (the automaton is deterministic (Johansson et $a l ., 1999 b)$ ), one may then say that the automaton is initially well-posed. This definition allows a situation in which a transition from location 1 to location 2 is immediately followed by a transition back to location 1 and so on. Hence, an infinite loop occurs with $\tau_{i}=\tau_{i}^{\prime}$ for all $i \geq i_{0}$ for some $i_{0}$ in the definition of an execution of the hybrid automaton (livelock). In (Lygeros et al., 1999), it is stated that for an initially well-posedness hybrid automaton "it still remains to be investigated whether executions can be extended over arbitrary time horizons." A first step to a stronger notion is obtained by requiring that a solution exists on some interval $[0, \varepsilon)$ with $\varepsilon>0$; system descriptions for which such solutions exist and are unique are called locally wellposed. In computer science terminology, such systems "allow time to progress". Finally, if solutions exist and are unique on the whole half-line $[0, \infty)$, then one speaks of global well-posedness.

\section{EXAMPLES}

In this section we will discuss several examples of hybrid systems, which will highlight the difference between the solution concepts mentioned above.

Example 5.1. The example of three balls in which inelastic impacts are modeled by successions of simple impacts (Figure 1) has also been mentioned in (Heemels et al., 1999) as an example of a system with live-lock, i.e. an infinite number of events at one time-instant. Suppose the balls all have unit mass and are touching at time 0 . The initial velocity $v_{1}(0)$ of ball 1 is equal to 1 and for balls 2 and $3 v_{2}(0)=v_{3}(0)=0$. By modelling all impacts separately, first an inelastic collision occurs between ball 1 and 2 resulting in $v_{1}(0+)=v_{2}(0+)=$ $\frac{1}{2}, v_{3}(0+)=0$. Next, ball 2 hits ball 3 resulting in $v_{1}(0++)=\frac{1}{2}, v_{2}(0++)=v_{3}(0++)=\frac{1}{4}$ after which ball 1 hits ball 2 again. In this way, a sequence of jumps is generated

$$
\begin{array}{llllllll}
v_{1}: & 1 & \frac{1}{2} & \frac{1}{2} & \frac{3}{8} & \frac{3}{8} & \frac{11}{32} & \ldots \\
v_{2}: & 0 & \frac{1}{2} & \frac{1}{4} & \frac{3}{8} & \frac{5}{16} & \frac{11}{32} \ldots \\
v_{3}: & 0 & 0 & \frac{1}{4} & \frac{1}{4} & \frac{5}{16} & \frac{5}{16} & \ldots
\end{array}
$$

which converges to $\left(\frac{1}{3}, \frac{1}{3}, \frac{1}{3}\right)^{\top}$ after which a smooth continuation is possible with constant and equal velocity for all balls. Note that a limiting operation is needed to get beyond the Zeno-time 0 . This might be considered a form of regularization, which works in the case the discrete state is subordinate to the continuous state. Other forms of regularization are discussed in (Lygeros et al., 1999; Johansson et al., 1999b) (cf. Section 6 below).

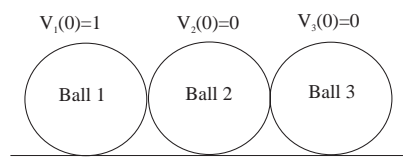

Fig. 1. Three balls example.

Several hybrid examples are around in the literature displaying right-accumulations of event times like the bouncing ball (Brogliato, 1996), the water tank system of the Introduction, and so on. Here we will present an example that fits into many of the mentioned model classes.

Example 5.2. The time reversed version of a system studied by Filippov (Filippov, 1988, p. 116) is given by

$$
\begin{aligned}
& \dot{x}_{1}=-\operatorname{sgn}\left(x_{1}\right)+2 \operatorname{sgn}\left(x_{2}\right) \\
& \dot{x}_{2}=-2 \operatorname{sgn}\left(x_{1}\right)-\operatorname{sgn}\left(x_{2}\right),
\end{aligned}
$$


where "sgn" denotes the signum-function given by $\operatorname{sgn}(x)=1$, if $x>0, \operatorname{sgn}(x)=-1$, if $x<0$ and $\operatorname{sgn}(x) \in[-1,1]$ when $x=0$ (note that this choice complies with Filippov's convex and Utkin's control equivalent definition). Solutions of this piecewise constant system are spiraling towards the origin, which is an equilibrium. Since $\frac{\mathrm{d}}{\mathrm{d} t}\left(\left|x_{1}(t)\right|+\left|x_{2}(t)\right|\right)=-2$, when $x(t) \neq 0$, solutions reach the origin in finite time. See Figure 2 for a trajectory. However, solutions cannot arrive at the origin without going through an infinite number of mode transitions (relay switches). Since these mode switches occur in a finite time interval, the event times contain a right-accumulation point (i.e. the time that the solution reaches the origin) after which the solution stays at zero. Again a limiting operation can define a "reasonable" solution beyond the Zeno point.

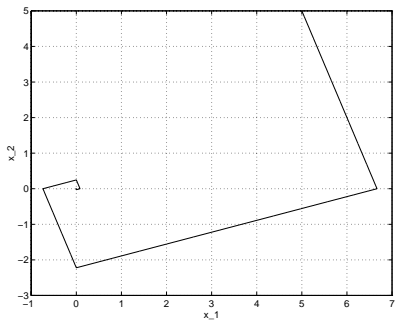

Fig. 2. Trajectory in the phase plane.

Left-accumulation points are awkward as well. Of course, time-reversal of systems with right-accumulations lead to such systems. Here, we mention an example that is discussed in (Pogromsky et al., 2001).

Example 5.3. Consider the following system

$$
\begin{gathered}
\dot{x}=\left(\begin{array}{lll}
0 & 1 & 0 \\
0 & 0 & 1 \\
0 & 0 & 0
\end{array}\right) x+\left(\begin{array}{l}
0 \\
0 \\
1
\end{array}\right) u \\
y=\left(\begin{array}{ccc}
1 & 0 & 0
\end{array}\right) x \\
u=-\operatorname{sgn} y .
\end{gathered}
$$

This system might also be written as the piece-wise linear bi-modal system:

$$
\begin{cases}\text { mode } 1: \dot{x}=A_{1} x, & \text { if } y=C x \geq 0 \\ \text { mode } 2: \dot{x}=A_{2} x, & \text { if } y=C x \leq 0\end{cases}
$$

where $x \in \mathbb{R}^{n}, y \in \mathbb{R}^{1}$ and

$$
\begin{gathered}
A_{1}=\left(\begin{array}{cccc}
0 & 1 & 0 & 0 \\
0 & 0 & 1 & 0 \\
0 & 0 & 0 & -1 \\
0 & 0 & 0 & 0
\end{array}\right), \quad A_{2}=\left(\begin{array}{llll}
0 & 1 & 0 & 0 \\
0 & 0 & 1 & 0 \\
0 & 0 & 0 & 1 \\
0 & 0 & 0 & 0
\end{array}\right) \\
C=\left(\begin{array}{llll}
1 & 0 & 0 & 0
\end{array}\right)
\end{gathered}
$$

There are infinitely many 'solutions' starting in the origin, if left-accumulations of relay switching times are allowed in the solution concept (as e.g. for Filippov's solution concept).

\section{COMPARISON}

\subsection{Use of event sets}

In a classification of solution concepts, one might begin by making a distinction as to whether or not there is explicit mention of different modes and/or an event set. Clearly, the execution for hybrid automata, the extended Carathéodory solutions, the forward and hybrid solution concept for complementarity systems mention the event set and the different modes. The solution concepts based on differential inclusions like
Filippov, Utkin or Euler solutions or the $L_{2}$-solution concept for complementarity systems are not using any references to modes or event times. In general this means that they allow broader types of event times sets. Therefore, a further classification can be made on the basis of the nature of the event set.

\subsection{Nature of event times set}

Definition 6.1. A set $\mathcal{E} \subset \mathbb{R}_{+}$is called an admissible event times set if it is closed and countable, and $0 \in \mathcal{E}$. To each admissible event times set $\mathcal{E}$, we associate a collection of intervals between events $\tau_{\mathscr{E}}=\left\{\left(t_{1}, t_{2}\right) \subset\right.$ $\mathbb{R}_{+} \mid t_{1}, t_{2} \in \mathscr{E} \cup\{\infty\}$ and $\left.\left(t_{1}, t_{2}\right) \cap \mathcal{E}=\emptyset\right\}$.

Note that both left and right accumulations of event times are allowed by the above definition.

Definition 6.2. An admissible event times set $\mathscr{E}$ is said to be left (right) Zeno free, if it does not contain any left (right) accumulation points.

Note that a hybrid time trajectory as defined in subsection 3.1 does not allow left-accumulation points. The event set $\mathscr{E}:=\{0\} \cup\left\{\frac{1}{n} \mid n \in \mathbb{N}\right\}$ and the corresponding sequence of intervals $\tau_{\varepsilon}$ cannot be rewritten in terms of a hybrid time trajectory. The hybrid time trajectory corresponds to a well-ordered event set with respect to the usual (increasing) ordering of the real line.

For general hybrid automata it is in principle impossible to define a solution concept beyond a rightaccumulation point of event times. In (Johansson et $a l ., 1999 a$ ) one mentions three possibilities of extension of Zeno executions: regularization (i.e. modifying the original Zeno automaton by adding temporal or spatial regularization parameters), averaging (averaging the vector field close to the Zeno time) and Filippov relaxation, which only applies in particular cases of differential equations with discontinuous right-hand sides. In principle, in the latter case we are dealing with a situation in which the location (discrete state) is determined fully by the continuous state. Actually, this is also true for solution concepts with no explicit mention of the discrete state or an application to classes like discontinuous differential equations, complementarity systems, or multi-modal linear systems as one can continue by taking the left-limit of the state - provided it exists - and define a continuation from the left-limit state (as if it was a new initial state). In this manner, a 'natural relaxation' is obtained.

Execution of hybrid automaton : $\&$ is left Zeno free. No continuation is possible beyond right-accumulation (besides regularization).

Filippov's, Euler or Utkin's : No restrictions on $\&$

Extended Carathéodory : $\&$ is left Zeno free. Continuation beyond right-accumulation point possible ${ }^{6}$ due to taking left-limit of state which can determine new discrete state.

Forward solution : $\&$ is left Zeno free. Continuation beyond right-accumulation point possible due to taking left-limit of state which can determine the new discrete state.

$L_{2}$-solution : No restrictions on $\mathcal{E}$.

6 In (Imura, 2001) a solution concept has been introduced based on a different mode transition rule called the "switch-based rule," which differs from the usually applied "mode-based rule." In this modified version of the extended Carathédory notion of solutions continuation beyond right-accumulation points is in principle not possible. 
Hybrid solution : No restrictions on $\mathbb{E}$.

For livelock the situation is similar as for continuation beyond right-accumulation points. This means that for Example 5.1 no local solution exists if taking limits of the event states at $t=0$ is not included in the solution concept. For solution concepts inferred from model classes in which the discrete state is determined by the continuous state, a local and even global solution can be defined for the three balls example. Similar statements hold for Example 5.2 for global solutions.

On the basis of the previous we can also state that Example 5.3 (see (Pogromsky et al., 2001)) will have one execution (for a hybrid automaton with three modes (including the sliding mode)), infinitely many Filippov solutions, no extended Carathéodory solution, one forward solution, infinitely many $L_{2}$-solutions and hybrid solutions.

The above discussion also indicates that time-reversal of executions, forward solutions, extended Carathéodory solutions does not necessarily yield such solutions again because of the well-ordered event times sets. Hence, the method of reversing time as is often used for analysis of smooth dynamical systems, is not possible without switching to a different (more general) solution concept.

\subsection{Simple variable structure system}

Consider the rather simple system

$$
\dot{x}=\left\{\begin{array}{ll}
1, & x>0 \\
-1, & x<0
\end{array},\right.
$$

which is of the form (1). It can easily be seen, that this system has infinitely many Filippov solutions (one can leave the sliding mode at each time instant) starting in the origin and satisfying $\dot{x} \in \operatorname{sgn}(x)$. However, it only has two extended Carathéodory solutions with $x(0)=0$, namely $x(t)=t$ and $x(t)=-t$. With respect to Euler solutions it depends on how the differential inclusion $\dot{x}=F(t, x)$ is constructed. Especially, the set-value of $F(t, 0)$ is crucial. E.g. if $F(t, 0)=\{0\}$ for all $t$ we only have one Euler solution (being zero) and if $F(t, 0)=\{1\}$ for all $t$ we have $x(t)=t$ as the only Euler solution starting in the origin. If we take $F(t, 0)=[-1,1]$ for all $t$ then we have a similar situation as for Filippov's solutions. However, taking time-varying $F(t, x)$ leads again to different situations as can be verified easily.

The time-reversed system (with a choice made at the switching surface by $F(0)=\{1\})$ (Clarke et al., 1998, Ex. 1.6(b)))

$$
\dot{x}= \begin{cases}-1, & x>0 \\ 1, & x \leq 0\end{cases}
$$

has for initial state $x(0)=0$ one Euler solution, no extended Carathéodory, and one Filippov solution. Note that extended Carathéodory solutions do not allow "sliding modes."

A final statement can be given on the difference between Filippov's convex and Utkin's control equivalent solutions, which can be shown to differ only by the dynamics exhibited at the sliding mode (see (van der Schaft and Schumacher, 1999, Ex. 3.2.1) for an example). Actually, it can be shown (Filippov, 1988) that under certain conditions the solution concepts by Filippov, Utkin and Aizerman / Pyatnitskii coincide.

\section{CONCLUSIONS}

In this paper we compared several solution concepts, which have been used for different hybrid model classes. The explicit mention of modes and / or event times and the kind of event times set that are allowed formed the major differences between the notions. The absence of accumulations of event times will cause many of the mentioned solution concepts to coincide. Also the kind of relaxations used (e.g. to go from a differential equation to a differential inclusion) affects the kind of solution trajectories obtained. The interaction between the solution concepts and the well-posedness issue has been pointed out. In a broad solution space existence of trajectories is easier satisfied than in a small one, for uniqueness the converse statement hold. One might also try to show existence of trajectories in a small class (e.g. forward solution) and show uniqueness in a bigger one (e.g. $L_{2}$ - or Filippov solutions). In this way, it is possible to obtain conditions that exclude particular types of Zeno behaviour (in this case left-accumulation points within the class of Filippov solutions) as is desirable from an analysis point of view as demonstrated by the two-tank system in the introduction.

\section{REFERENCES}

Alur, R. and T.A. Henzinger (1997). Modularity for timed an hybrid systems. In: CONCUR 97. Lect. Notes Comp. Sc. 1243. pp. 7488.

Alur, R., C. Courcoubetis, N. Halbwachs, T. A. Henzinger, P.-H. Ho, X. Nicollin, A. Olivero, J. Sifakis and S. Yovine (1995). The algorithmic analysis of hybrid systems. Theoretical Computer Science 138, 3-34.

Aubin, J.-P. and A. Cellina (1984). Differential Inclusions: Set-Valued Maps and Viability Theory. Springer. Berlin.

Brogliato, B. (1996). Nonsmooth Impact Mechanics. Models, Dynamics and Control. Vol. 220 of Lecture Notes in Control and Information Sciences. Springer. London.

Clarke, F.H., Yu. S. Ledyaev, E.D. Sontag and A.I. Subbotin (1997). Asymptotic controllability implies feedback stabilization. IEEE Trans. Automat. Contr. 42, 1394-1407.

Clarke, F.H., Yu. S. Ledyaev, R.J. Stern and P.R. Wolenski (1998). Nonsmooth Analysis and Control Theory. Springer. Berlin.

Filippov, A.F. (1988). Differential Equations with Discontinuous Righthand Sides. Mathematics and Its Applications. Kluwer. Dordrecht, The Netherlands.

Heemels, W.P.M.H., J.M. Schumacher and S. Weiland (1999). Wellposedness of linear complementarity systems. In: Proc. CDC Phoenix (USA). pp. 3037-3042.

Heemels, W.P.M.H., J.M. Schumacher and S. Weiland (2000). Linear complementarity systems. SIAM J. Appl. Math. 60, 1234-1269.

Imura, J.-I. and A.J. van der Schaft (2000). Characterization of wellposedness of piecewise linear systems. IEEE Trans. Aut. Contr. 45(9), 1600-1619.

Imura, J.-I. (2001). Well-posedness analysis of switch-driven piecewise affine systems. Submitted to IEEE Trans. Aut. Contr. Preliminary versions at NOLCOS'O1 and ACC'O1.

Johansson, K.H., J. Lygeros, S. Sastry and M. Egerstedt (1999a). Simulation of Zeno hybrid automata. In: Proc. CDC in Phoenix (USA). pp. 3538-3543.

Johansson, K. H., M. Egerstedt, J. Lygeros and S. Sastry (1999b). On the regularization of zeno hybrid automata. Systems and Control Letters 38, 141-150.

Lygeros, J., K.H. Johansson, S. Sastry and M. Egerstedt (1999). On the existence and uniqueness of executions of hybrid automata. In: Proc. CDC Phoenix (USA). pp. 2249-2254.

Lynch, N., R. Segala, F. Vaandrager and H.B. Weinberger (1996). Hybrid I/O automata. In: Hybrid Systems III. Springer. Berlin. Lect. Notes Comp. Sci., vol 1066.

Pogromsky, A.Yu., W.P.M.H. Heemels and H. Nijmeijer (2001). On well-posedness of relay systems. In: Proceedings of NOLCOS 2001. St. Petersburg (Russia).

Utkin, V.I. (1981). Sliding Regimes in Optimization and Control Problems. Nauka. Moscow.

van der Schaft, A.J. and J.M. Schumacher (1999). An Introduction to Hybrid Dynamical Systems. Vol. 251 of Lecture Notes in Control and Information Sciences. Springer. London.

van der Schaft, A. J. and J. M. Schumacher (1998). Complementarity modeling of hybrid systems. IEEE Trans. Automat. Contr. AC43, 483-490. 